\title{
Untreated newly diagnosed essential hypertension is associated with nonalcoholic fatty liver disease in a population of a hypertensive center
}

This article was published in the following Dove Press journal:

Clinical and Experimental Gastroenterology

13 January 2016

Number of times this article has been viewed

\author{
Spyros Michopoulos' \\ Vasiliki I Chouzouri' \\ Efstathios D Manios' \\ Eirini Grapsa² \\ Zoi Antoniou' \\ Christos A Papadimitriou ${ }^{3}$ \\ Nikolaos Zakopoulos' \\ Athanasios-Meletios \\ Dimopoulos' \\ 'Department of Clinical Therapeutics, \\ Medical School of Athens, Alexandra \\ Hospital, Athens, Greece; ${ }^{2}$ Nephrology \\ Department, Medical School of \\ Athens, Aretaieio Hospital, Athens, \\ Greece; ${ }^{3}$ Oncology Department, \\ Medical School of Athens, Aretaieio \\ Hospital, Athens, Greece
}

Correspondence: Vasiliki I Chouzouri Department of Clinical Therapeutics, Medical School of Athens, Alexandra Hospital, Kleovoulis 4, Zografou I5773, Athens, Greece

Tel +302107788180

$\mathrm{Fax}+302132162889$

Email vickychouzouri@gmail.com
Purpose: Recent studies have demonstrated that hypertension (HTN) is associated with nonalcoholic fatty liver disease (NAFLD) in treated hypertensive patients. The aim of this study was to investigate the association between newly diagnosed essential HTN and NAFLD in untreated hypertensive patients.

Patients and methods: A consecutive series of 240 subjects (143 hypertensives and 97 normotensives), aged 30-80 years, without diabetes mellitus were enrolled in the study. Subjects with 24-hour systolic blood pressure (SBP) values $\geq 130 \mathrm{mmHg}$ and/or diastolic BP values $\geq 80 \mathrm{mmHg}$ were defined as hypertensives. NAFLD was defined as the presence of liver hyperechogenicity on ultrasound.

Results: Body mass index $(P=0.002)$ and essential HTN $(P=0.016)$ were independently associated with NAFLD in the multivariate logistic regression model. Furthermore, the multivariate analysis revealed that morning SBP $(P=0.044)$ was independently associated with NAFLD.

Conclusion: Untreated, newly diagnosed essential HTN is independently associated with NAFLD. Ambulatory BP monitoring could be used for the diagnosis of essential HTN in patients with NAFLD.

Keywords: ambulatory blood pressure, liver steatosis, hypertension, nonalcoholic fatty liver disease, body mass index

\section{Introduction}

Nonalcoholic fatty liver disease (NAFLD) is the most common liver disease worldwide. Its prevalence is estimated to be up to $30 \%$ in developed countries and nearly $10 \%$ in developing nations. ${ }^{1}$ NAFLD includes a wide spectrum of liver injury ranging from mild liver steatosis to nonalcoholic steatohepatitis (NASH), advanced fibrosis, and progression to cirrhosis and hepatocellular cancer. ${ }^{1,2}$ Subjects with NAFLD should be closely monitored and preventive lifestyle modifications should be implemented to avoid progression of the benign steatosis to NASH and fibrosis. ${ }^{3}$ Cardiovascular disease and liver injury associated with cirrhosis and its complications are two of the most important causes of death in NAFLD patients. ${ }^{4}$ Moreover, NAFLD is associated with coronary artery disease, cerebrovascular disease and peripheral vascular disease, and arterial hypertension (HTN). ${ }^{5-7}$

Arterial HTN, as a component of metabolic syndrome, seems to be a predictor of NAFLD. ${ }^{8-12}$ More specifically, Vasunta et $\mathrm{al}^{11}$ showed that patients with fatty liver had significantly higher ambulatory daytime and nighttime systolic blood pressure (SBP) levels than those with normal liver. Moreover, Bedogni et al, ${ }^{12}$ in the Dionysos nutrition and liver study, concluded that HTN was independently associated with NAFLD in patients 
under antihypertensive treatment. Additionally, treatment of essential HTN with angiotensin-converting-enzyme inhibitors and angiotensin II receptor blockers seems to improve steatosis. ${ }^{13-17}$ However, approximately $50 \%$ of the subjects who were included in the previous studies were under unknown hypertensive treatment.

As far as we are concerned, there is limited information about the association of untreated newly diagnosed essential HTN using ambulatory blood pressure (BP) monitoring recordings with NAFLD. The aim of our study was to investigate the possible association between newly diagnosed essential HTN using ambulatory BP monitoring recordings and NAFLD in never treated hypertensive patients.

\section{Patients and methods}

From November 2009 to June 2012, we took detailed medical histories from all patients (1,771 in total) who were referred to the hypertensive center of our hospital. A number of exclusion criteria were defined in order to minimize the possibility of interaction with other diseases. Patients with documented diagnosis of malignancy, chronic liver disease (autoimmune hepatitis, hemochromatosis, etc), positive serum markers for hepatitis $\mathrm{B}$ or $\mathrm{C}$, alcohol consumption over $30 \mathrm{~g} / \mathrm{d}$ for men and $20 \mathrm{~g} / \mathrm{d}$ for women, diabetes mellitus (fasting glucose $>126 \mathrm{mg} / \mathrm{dL}$ ), secondary HTN, and estimated glomerular filtration rate $^{18}(\mathrm{eGFR})<60 \mathrm{~mL} /$ $\min / 1.73 \mathrm{~m}^{2}$ were excluded from the study. Also, patients taking antihypertensive treatment, corticosteroids, tricyclic antidepressants, amiodarone, tetracyclines, chronic salicylates, and other medications ${ }^{19}$ causing deposition of fat at the liver were excluded.

All subjects underwent office BP measurements, 24-hour ambulatory BP monitoring, liver ultrasound, and blood test examination. Body mass index (BMI) was calculated by dividing weight $(\mathrm{kg})$ by the square of height $\left(\mathrm{m}^{2}\right) .{ }^{20}$ Smoking status was also recorded. Alcohol consumption was defined as grams of absolute alcohol consumed per day. The waist circumference was measured using a tape which was placed to the nearest $0.5 \mathrm{~cm}$ between the last rib and the iliac crest while the person was standing with feet approximately $25-30 \mathrm{~cm}$ apart and at the end of a normal expiration. The measurer stood beside the person and applied the tape snugly, without compressing any underlying soft tissues. ${ }^{21}$

A total of 240 patients fulfilled the inclusion criteria and were enrolled in the study. The patients were divided into two groups according to the results of the liver ultrasound. Those with hyperechogenicity of liver in contrast to the cortical portion of the right kidney, or either vascular blurring or deep attenuation were labeled as the group with NAFLD and those without any of the above characteristics on ultrasound were labeled as the group of control patients. ${ }^{22}$ The study population was divided into two groups according to 24-hour mean ambulatory BP values: patients with elevated 24-hour SBP $\geq 130 \mathrm{mmHg}$ and/or diastolic BP (DBP) values $\geq 80 \mathrm{mmHg}$ were defined as hypertensives and those with normal 24-hour BP values $(<130 / 80 \mathrm{mmHg})$ were defined as normotensives. ${ }^{23}$ The patients were also divided into two groups: those with white-coat HTN and those without white-coat HTN. White-coat HTN is present when office SBP is $>140 \mathrm{mmHg}$ or office DBP $>90 \mathrm{mmHg}$ and daytime average SBP is $<135 \mathrm{mmHg}$ and DBP $<85 \mathrm{mmHg} .{ }^{24}$

All participants provided informed written consent, and the study was approved by the local scientific committee of Alexandra Hospital.

\section{BP measurements}

Office BP was measured by a physician in both arms using a clinically validated automated sphygmomanometer (Omron 705IT; Omron Healthcare, Kyoto, Japan). The patients were seated for 5 minutes in a quiet room before beginning BP measurements. Individuals with differences in BP between the arms greater than $20 \mathrm{mmHg}$ for SBP and $10 \mathrm{mmHg}$ for DBP were excluded from the study $(n=3)$. During the measurements, the participants' arm was supported and placed at the level of the heart. Three BP measurements taken at a 1-minute interval were averaged to obtain a single systolic and diastolic office BP value.

Ambulatory BP measurements were recorded using oscillometric Spacelabs 90207 equipment (Spacelabs, Redmond, WA, USA). The daytime and nighttime periods were identified by using the fixed-narrow time interval approach, recommended by the European Society of Hypertension. ${ }^{25}$ Daytime readings ( 9 am-9 pm) and morning BP measurements ( 6 am-9 am) were taken every 15 minutes, and nighttime readings were taken ( 1 am-6 am) every 30 minutes. The retiring period between $9 \mathrm{pm}$ and 1 am is eliminated in order to avoid variations in the time spent in bed that may exist between patients of different ages. All patients were instructed to rest and sleep during the nighttime and to maintain their usual activities during the day. None of the study participants were bedridden or hospitalized during ambulatory BP monitoring. Individuals who reported that they had not rested during the night interval were excluded from further evaluation $(n=4)$. Individuals who reported unusual events, for example, unusual physical or emotional events, during the day were excluded from the study $(n=3)$. 
If the examination had less than $70 \%$ of the expected number of valid values because of frequent artifacts, then the examination was repeated.

\section{Laboratory data}

Blood sample was taken from all the participants after overnight fast. Complete blood account, glucose serum fast (GLU), cholesterol (CHOL), triglycerides (TRIGL), highdensity lipoprotein (HDL), low-density lipoprotein (LDL), aspartate and alanine aminotransferases (AST, ALT), gamma glutamyl transpeptidase (GGT), alkaline phosphatase (ALP), urea nitrogen, serum creatinine, and high-density c-reactive protein (Hs-CRP) were assessed. For the biochemical analysis, Integra 800, spectrophotometric analyzer (Roche, Basel, Switzerland) was used. For the measurement of Hs-CRP, BN2-Nephelometer II, Siemens analyzer (Siemens, Berlin and Munich, Germany) was used.

\section{Liver ultrasound}

The ultrasonographic diagnosis (probe: convex, $4 \mathrm{MHz}$; Envisor, Philips, Holland, the Netherlands) of steatosis was made by the same operator, who was blinded regarding the BP levels and the laboratory findings of the patients. Subjects were labeled as having liver steatosis when there was hyperechogenicity of liver in contrast to the cortical portion of the right kidney, and either vascular blurring or deep attenuation. ${ }^{22}$

\section{Statistical analyses}

The Kolmogorov-Smirnov test was used to assess the normality of continuous variables. Continuous variables are expressed as mean \pm standard deviation, while categorical variables are expressed as relative frequencies (\%). The variables office SBP, eGFR, and Hs-CRP had skewed distribution and were transformed. More specifically, eGFR was transformed using square root. Office SBP and Hs-CRP were logarithmically transformed. Chi-square was used to explore the relationship between categorical variables. Independent-samples $t$-test was used to investigate the difference between normally distributed data accordingly. Pearson product-moment correlation was conducted in order to investigate correlations between continuous variables. Continuous variables with a correlation coefficient higher than 0.7 were not included in the same model. So, BMI and waist circumference were not included in the same model. In the initial univariate analyses, a threshold of $P<0.1$ (because of the risk of a type II error because of low statistical power in such an analysis) was used to identify candidate variables for inclusion in the final model. Multiple logistic regression analysis was conducted in order to identify variables independently associated with essential HTN and NAFLD. Statistical significance was achieved with a two-tailed value of $P<0.05$. SPSS software (SPSS 15.0 version, Chicago, IL, USA) was used for statistical analysis.

\section{Results}

The mean age was $54 \pm 11$ years for those with NAFLD and $55 \pm 11$ for those with normal liver. Hypertensives constitute $59.6 \%$ of the whole sample. NAFLD was detected in $67.9 \%$ of the study population. The characteristics of subjects with NAFLD and those without NAFLD are shown in Table 1.

Table I Characteristics of patients with and without nonalcoholic fatty liver disease

\begin{tabular}{|c|c|c|c|}
\hline Variables & $\begin{array}{l}\text { NAFLD } \\
\text { patients } \\
(\mathrm{N}=163)\end{array}$ & $\begin{array}{l}\text { Controls } \\
(\mathrm{N}=77)\end{array}$ & $P$-value \\
\hline Age (years) & $54 \pm 11$ & $55 \pm 11$ & 0.470 \\
\hline Male (\%) & 36.2 & 28.6 & 0.244 \\
\hline Waist circumference $(\mathrm{cm})$ & $103 \pm 12$ & $88 \pm 11$ & $<0.001$ \\
\hline $\operatorname{BMI}\left(\mathrm{kg} / \mathrm{m}^{2}\right)$ & $30 \pm 5.5$ & $25.64 \pm 3.8$ & $<0.001$ \\
\hline Smoking (\%) & 27.1 & 23.5 & 0.584 \\
\hline eGFR $\left(\mathrm{mL} / \mathrm{min} / \mathrm{l} .73 \mathrm{~m}^{2}\right)$ & $97 \pm 27$ & $101 \pm 23$ & 0.399 \\
\hline Hypertension (\%) & 65.6 & 46.8 & 0.008 \\
\hline Office SBP (mmHg) & $149 \pm 17$ & $145 \pm 16$ & 0.070 \\
\hline Office DBP (mmHg) & $90 \pm 11$ & $88 \pm 10$ & 0.160 \\
\hline 24-hour mean SBP (mmHg) & $13 \mid \pm 12$ & $126 \pm 12$ & 0.002 \\
\hline 24-hour mean DBP (mmHg) & $80 \pm 9.50$ & $78 \pm 9.11$ & 0.104 \\
\hline Daytime mean SBP (mmHg) & $136 \pm 13.04$ & $|3| \pm 12.87$ & 0.006 \\
\hline Daytime mean DBP $(\mathrm{mmHg})$ & $85 \pm 10$ & $83 \pm 9.8$ & 0.182 \\
\hline Nighttime mean SBP (mmHg) & $120 \pm 14.00$ & $113.4 \pm 18.00$ & 0.004 \\
\hline Nighttime mean DBP ( $\mathrm{mmHg}$ ) & $7 I \pm 11$ & $69 \pm 10$ & 0.284 \\
\hline Morning mean SBP $(\mathrm{mmHg})$ & $13 \mid \pm 14$ & $125 \pm 17$ & 0.013 \\
\hline Morning mean DBP $(\mathrm{mmHg})$ & $8 I \pm 11$ & $79 \pm 11$ & 0.153 \\
\hline White-coat hypertension (\%) & 20.9 & 26 & 0.435 \\
\hline AST (IU/L) & $22 \pm 9.70$ & $|9.10 \pm 5.5|$ & 0.023 \\
\hline ALT (IU/L) & $29.3 \pm 23$ & $22.40 \pm 21$ & 0.001 \\
\hline GGT (IU/L) & $24.8 \pm 16.3$ & $15.6 \pm 8.2$ & $<0.001$ \\
\hline ALP (IU/L) & $97.41 \pm 105.44$ & $81.33 \pm 47.23$ & 0.238 \\
\hline $\mathrm{CHOL}(\mathrm{mg} / \mathrm{dL})$ & $217 \pm 39$ & $210 \pm 42$ & 0.243 \\
\hline $\mathrm{LDL}(\mathrm{mg} / \mathrm{dL})$ & $138 \pm 36$ & $136 \pm 40$ & 0.729 \\
\hline HDL (mg/dL) & $56.34 \pm 19.85$ & $63.60 \pm 17.36$ & 0.011 \\
\hline TRIGL (mg/dL) & $130 \pm 65$ & $94 \pm 51$ & $<0.001$ \\
\hline $\mathrm{Hs}-\mathrm{CRP}(\mathrm{mg} / \mathrm{L})$ & $1.32 \pm 2.32$ & $0.84 \pm 1.29$ & 0.057 \\
\hline $\mathrm{GLU}(\mathrm{mg} / \mathrm{dL})$ & $95.66 \pm 12.52$ & $90.89 \pm 8.77$ & 0.001 \\
\hline
\end{tabular}

Notes: Noncontinuous variables are given as percentages. Continuous variables are presented as mean $\pm S D$. Upper reference limits are $45 \mathrm{IU} / \mathrm{L}$ for $A S T, 45 \mathrm{IU} / \mathrm{L}$ for ALT, $55 \mathrm{IU} / \mathrm{L}$ for GGT, I $50 \mathrm{IU} / \mathrm{L}$ for ALP, $200 \mathrm{mg} / \mathrm{dL}$ for CHOL, $140 \mathrm{mg} / \mathrm{dL}$ for LDL, $160 \mathrm{mg} / \mathrm{dL}$ for TRIGL, $5 \mathrm{mg} / \mathrm{L}$ for Hs-CRP, and I $10 \mathrm{mg} / \mathrm{dL}$ for GLU in our laboratory. Lower reference limit is $35 \mathrm{mg} / \mathrm{dL}$ for HDL in our laboratory.

Abbreviations: NAFLD, nonalcoholic fatty liver disease; BMI, body mass index; eGFR, estimated glomerular filtration rate; SBP, systolic blood pressure DBP, diastolic blood pressure; AST, aspartate aminotransferase; ALT, alanine aminotransferase; GGT, gamma glutamyl transpeptidase; ALP, alkaline phosphatase; $\mathrm{CHOL}$, cholesterol; LDL, low-density lipoprotein; HDL, high-density lipoprotein; TRIGL, triglycerides; Hs-CRP, high-density c-reactive protein; GLU, glucose serum fast; SD, standard deviation. 
Subjects with NAFLD showed higher prevalence of essential HTN than those without NAFLD. Five of 77 patients without steatosis and seven of 163 patients with NAFLD were on lipid-lowering treatment with statins $(P=0.696)$. Those with NAFLD presented higher values of BMI, waist circumference, AST, ALT, GGT, TRIGL, and glucose than those without NAFLD. On the other hand, those with fatty liver had lower levels of HDL than those without fatty liver.

The levels of office SBP and DBP did not differ between the two groups. Additionally, the values of mean DBP during all day periods (24 hours, daytime, nighttime, and morning) did not differ between the two groups. The levels of 24-hour $(P=0.002)$, daytime $(P=0.006)$, nighttime $(P=0.004)$, and morning $\operatorname{SBP}(P=0.013)$ were higher in those with fatty liver than in those without fatty liver. The percentage of white-coat HTN did not differ in those with NAFLD compared to those without NAFLD $(P=0.435)$.

All statistically significant variables $(P<0.1)$ at univariate analysis were included in the multivariate logistic model. BMI (odds ratio [OR]: 1.224; 95\% confidence interval [CI]: 1.079-1.390; $P=0.002$ ) and essential HTN (OR: 3.584; 95\% CI: $1.274-10.076 ; P=0.016)$ were factors independently associated with fatty liver (Table 2$)$. GGT $(P=0.060)$ showed only a tendency. Furthermore, morning SBP $(P=0.044)$ was independently associated with NAFLD after adjustment for BMI, AST, ALT, GGT, HDL, TRIGL, GLU, CRP, and office SBP. Twenty-four-hour $(P=0.055)$ and nighttime $(P=0.077)$ SBP showed a tendency but was not associated with fatty liver after adjustment for BMI, AST, ALT, GGT, HDL, TRIGL, GLU, CRP, and office SBP, whereas daytime SBP $(P=0.109)$ was not associated with NAFLD at multivariate analysis. BMI and waist circumference were not included in the same model due to collinearity reasons.

The prevalence of essential HTN did not differ between men with NAFLD and men with normal liver $(P=0.321)$. Men with NAFLD had significantly higher waist circumference, BMI, and GGT values than men with normal liver. The levels of office SBP and DBP did not differ between two groups. Additionally, the values of mean SBP and DBP during all day periods (24 hours, daytime, nighttime, and morning) did not differ between the two groups (Table 3). BMI, AST, and GGT were associated significantly with NAFLD in the initial univariate analyses. In the multivariate logistic analysis, BMI (OR: 1.237; 95\% CI: 1.047-1.461; $P=0.012$ ) showed significant and independent association with NAFLD in men (Table 4). GGT (OR: 1.056; 95\% CI: 0.991-1.127; $P=0.093$ ) showed only a tendency. BMI and waist circumference were not included in the same model due to collinearity reasons.
Table 2 Variables associated with nonalcoholic fatty liver disease in whole sample

\begin{tabular}{|c|c|c|c|c|}
\hline Variables & B & OR & $95 \% \mathrm{Cl}$ & $P$-value \\
\hline \multicolumn{5}{|l|}{ Bivariate analysis } \\
\hline Age (years) & -0.010 & 0.990 & $0.963-1.017$ & 0.467 \\
\hline Sex (male) & 0.349 & 1.418 & $0.787-2.555$ & 0.245 \\
\hline Waist circumference $(\mathrm{cm})$ & 0.112 & 1.118 & $1.080-1.157$ & $<0.001$ \\
\hline $\mathrm{BMI}\left(\mathrm{kg} / \mathrm{m}^{2}\right)$ & 0.237 & 1.267 & $1.162-1.383$ & $<0.001$ \\
\hline Smoking (yes) & 0.188 & 1.207 & $0.618-2.359$ & 0.582 \\
\hline eGFR $\left(\mathrm{mL} / \mathrm{min} / \mathrm{l} .73 \mathrm{~m}^{2}\right)^{\mathrm{a}}$ & -0.080 & 0.923 & $0.739-1.154$ & 0.484 \\
\hline Hypertension (yes) & 0.778 & 2.177 & $1.253-3.780$ & 0.006 \\
\hline Office SBP $\left(\mathrm{mmHg}^{\mathrm{b}}\right.$ & 1.055 & 2.872 & $0.403-3.320$ & 0.074 \\
\hline Office DBP $(\mathrm{mmHg})$ & 0.018 & 1.018 & $0.993-1.044$ & 0.161 \\
\hline $\begin{array}{l}\text { 24-hour mean SBP } \\
(\mathrm{mmHg})\end{array}$ & 0.037 & 1.037 & $1.013-1.063$ & 0.003 \\
\hline $\begin{array}{l}\text { 24-hour mean DBP } \\
\text { (mmHg) }\end{array}$ & 0.025 & 1.026 & $0.995-1.057$ & 0.106 \\
\hline $\begin{array}{l}\text { Daytime mean SBP } \\
(\mathrm{mmHg})\end{array}$ & 0.031 & 1.032 & $1.009-1.055$ & 0.006 \\
\hline $\begin{array}{l}\text { Daytime mean DBP } \\
(\mathrm{mmHg})\end{array}$ & 0.019 & 1.019 & $0.991-1.048$ & 0.183 \\
\hline $\begin{array}{l}\text { Nighttime mean SBP } \\
(\mathrm{mmHg})\end{array}$ & 0.030 & 1.030 & $1.010-1.051$ & 0.004 \\
\hline $\begin{array}{l}\text { Nighttime mean DBP } \\
(\mathrm{mmHg})\end{array}$ & 0.015 & 1.015 & $0.988-1.042$ & 0.285 \\
\hline $\begin{array}{l}\text { Morning mean SBP } \\
(\mathrm{mmHg})\end{array}$ & 0.025 & 1.025 & $1.005-1.045$ & 0.013 \\
\hline $\begin{array}{l}\text { Morning mean DBP } \\
(\mathrm{mmHg})\end{array}$ & 0.019 & 1.019 & $0.993-1.046$ & 0.153 \\
\hline $\begin{array}{l}\text { White-coat hypertension } \\
\text { (yes) }\end{array}$ & -0.225 & 0.798 & $0.49 I-1.297$ & 0.463 \\
\hline AST (IU/L) & 0.060 & 1.062 & $1.012-1.115$ & 0.015 \\
\hline ALT (IU/L) & 0.023 & 1.024 & $1.001-1.046$ & 0.038 \\
\hline GGT (IU/L) & 0.081 & 1.084 & $1.044-1.125$ & $<0.001$ \\
\hline ALP (IU/L) & 0.004 & 1.004 & $0.998-1.009$ & 0.248 \\
\hline $\mathrm{CHOL}(\mathrm{mg} / \mathrm{dL})$ & 0.004 & 1.004 & $0.997-1.012$ & 0.243 \\
\hline $\mathrm{LDL}(\mathrm{mg} / \mathrm{dL})$ & 0.001 & 1.001 & $0.993-1.009$ & 0.728 \\
\hline HDL (mg/dL) & -0.020 & 0.980 & $0.965-0.996$ & 0.015 \\
\hline TRIGL (mg/dL) & 0.014 & 1.014 & $1.007-1.021$ & $<0.00$ I \\
\hline $\mathrm{Hs}-\mathrm{CRP}(\mathrm{mg} / \mathrm{L})^{\mathrm{c}}$ & 0.563 & 1.756 & $0.978-3.152$ & 0.060 \\
\hline GLU (mg/dL) & 0.042 & 1.043 & $1.013-1.074$ & 0.004 \\
\hline \multicolumn{5}{|l|}{ Multivariate logistic regression ${ }^{\mathrm{a}}$} \\
\hline BMI $\left(\mathrm{kg} / \mathrm{m}^{2}\right)$ & 0.203 & 1.224 & I.079-1.390 & 0.002 \\
\hline Hypertension (yes) & 1.276 & 3.584 & $1.274-10.076$ & 0.016 \\
\hline GGT (IU/L) & 0.046 & 1.047 & $0.998-1.099$ & 0.060 \\
\hline
\end{tabular}

Notes: Odds ratio and $95 \% \mathrm{Cl}$ adjusted by office SBP, AST, and all covariables with $P<0.100$ in bivariate analysis. BMI and waist circumference were not included in the same model because they are continuous variables with a correlation coefficient higher than 0.7. The same applies to 24-hour mean SBP and daytime mean SBP, 24hour mean SBP and nighttime mean SBP, and 24-hour mean SBP and morning mean SBP. Upper reference limits are $45 \mathrm{IU} / \mathrm{L}$ for AST, $45 \mathrm{IU} / \mathrm{L}$ for ALT, $55 \mathrm{IU} / \mathrm{L}$ for GGT, $150 \mathrm{IU} / \mathrm{L}$ for ALP, $200 \mathrm{mg} / \mathrm{dL}$ for CHOL, $140 \mathrm{mg} / \mathrm{dL}$ for LDL, $160 \mathrm{mg} / \mathrm{dL}$ for TRIGL, $5 \mathrm{mg} / \mathrm{L}$ for Hs-CRP, and I $10 \mathrm{mg} / \mathrm{dL}$ for GLU in our laboratory. Lower reference limit is $35 \mathrm{mg} / \mathrm{dL}$ for HDL in our laboratory. ${ }^{2}$ The variable eGFR was transformed using square root; b the variable office SBP was logarithmically transformed; 'the variable Hs-CRP was logarithmically transformed.

Abbreviations: $B$, regression coefficient; OR, odds ratio; $\mathrm{Cl}$, confidence interval; BMI, body mass index; eGFR, estimated glomerular filtration rate; SBP, systolic blood pressure; DBP, diastolic blood pressure; $\mathrm{AST}$, aspartate aminotransferase; ALT, alanine aminotransferase; GGT, gamma glutamyl transpeptidase; ALP, alkaline phosphatase; CHOL, cholesterol; LDL, low-density lipoprotein; HDL, high-density lipoprotein; TRIGL, triglycerides; Hs-CRP, high-density c-reactive protein; GLU, glucose serum fast. 
Table 3 Characteristics of men with and without nonalcoholic fatty liver disease

\begin{tabular}{|c|c|c|c|}
\hline Variables & $\begin{array}{l}\text { NAFLD } \\
\text { patients } \\
(\mathbf{N}=59)\end{array}$ & $\begin{array}{l}\text { Controls } \\
(\mathrm{N}=22)\end{array}$ & $P$-value \\
\hline Age (years) & $5 I \pm I I$ & $55 \pm 11$ & 0.100 \\
\hline Waist circumference (cm) & $106 \pm 12$ & $92 \pm 10$ & $<0.00 \mathrm{I}$ \\
\hline BMI $\left(\mathrm{kg} / \mathrm{m}^{2}\right)$ & $30 \pm 4.7$ & $26 \pm 4$ & $<0.00$ I \\
\hline Smoking (\%) & 32.7 & 27.8 & 0.695 \\
\hline eGFR $\left(\mathrm{mL} / \mathrm{min} / \mathrm{l} .73 \mathrm{~m}^{2}\right)$ & $80 \pm 2$ & $81 \pm 2$ & 0.802 \\
\hline Hypertension (\%) & 76.3 & 86.4 & 0.321 \\
\hline Office SBP (mmHg) & $153 \pm 19$ & $149 \pm 13$ & 0.290 \\
\hline Office DBP (mmHg) & $93 \pm 13$ & $91 \pm 8$ & 0.526 \\
\hline 24-hour mean SBP (mmHg) & $135 \pm 13$ & $13 \mid \pm 10$ & 0.168 \\
\hline 24-hour mean DBP (mmHg) & $84 \pm 11$ & $83 \pm 7$ & 0.716 \\
\hline Daytime mean SBP (mmHg) & $|4| \pm \mid 4$ & $137 \pm 9$ & 0.300 \\
\hline Daytime mean DBP $(\mathrm{mmHg})$ & $89 \pm 11$ & $88 \pm 7$ & 0.816 \\
\hline Nighttime mean SBP (mmHg) & $122 \pm 16$ & $119 \pm 12$ & 0.316 \\
\hline Nighttime mean DBP $(\mathrm{mmHg})$ & $73 \pm 11$ & $71 \pm 8$ & 1.000 \\
\hline Morning mean SBP $(\mathrm{mmHg})$ & $133 \pm 15$ & $13 \mid \pm 13$ & 0.549 \\
\hline Morning mean DBP $(\mathrm{mmHg})$ & $84 \pm 11$ & $83 \pm 7$ & 0.813 \\
\hline White-coat hypertension (\%) & 11.9 & 12.5 & 0.293 \\
\hline AST (IU/L) & $25 \pm 11$ & $20 \pm 7$ & 0.097 \\
\hline ALT (IU/L) & $39 \pm 31$ & $32 \pm 36$ & 0.439 \\
\hline GGT (IU/L) & $30 \pm 18$ & $21 \pm 12$ & 0.038 \\
\hline ALP (IU/L) & $93 \pm 55$ & $78 \pm 44$ & 0.796 \\
\hline $\mathrm{CHOL}(\mathrm{mg} / \mathrm{dL})$ & $220 \pm 39$ & $212 \pm 37$ & 0.265 \\
\hline LDL (mg/dL) & $148 \pm 46$ & $136 \pm 30$ & 0.205 \\
\hline $\mathrm{HDL}(\mathrm{mg} / \mathrm{dL})$ & $49 \pm 16$ & $56 \pm 16$ & 0.147 \\
\hline TRIGL (mg/dL) & $138 \pm 75$ & $109 \pm 50$ & 0.127 \\
\hline $\mathrm{Hs}-\mathrm{CRP}$ (mg/dL) & $1.25 \pm 1.75$ & $0.74 \pm 0.69$ & 0.314 \\
\hline GLU (mg/L) & $99 \pm 16$ & $93 \pm 8$ & 0.112 \\
\hline
\end{tabular}

Notes: Data are presented as mean \pm standard deviation. Upper reference limits are $45 \mathrm{IU} / \mathrm{L}$ for AST, $45 \mathrm{IU} / \mathrm{L}$ for ALT, $55 \mathrm{IU} / \mathrm{L}$ for GGT, $150 \mathrm{IU} / \mathrm{L}$ for ALP, $200 \mathrm{mg} /$ $\mathrm{dL}$ for CHOL, $140 \mathrm{mg} / \mathrm{dL}$ for LDL, $160 \mathrm{mg} / \mathrm{dL}$ for TRIGL, $5 \mathrm{mg} / \mathrm{L}$ for Hs-CRP, and $110 \mathrm{mg} / \mathrm{dL}$ for GLU in our laboratory. Lower reference limit is $35 \mathrm{mg} / \mathrm{dL}$ for HDL in our laboratory.

Abbreviations: NAFLD, nonalcoholic fatty liver disease; eGFR, estimated glomerular filtration rate; BMI, body mass index; SBP, systolic blood pressure; DBP, diastolic blood pressure; AST, aspartate aminotransferase; ALT, alanine aminotransferase; GGT, gamma glutamyl transpeptidase; ALP, alkaline phosphatase; $\mathrm{CHOL}$, cholesterol; LDL, low-density lipoprotein; HDL, high-density lipoprotein; TRIGL, triglycerides; Hs-CRP, high-density c-reactive protein; GLU, glucose serum fast.

Women with NAFLD (75.8\%) had significantly $(P=0.001)$ higher prevalence of essential HTN than those with normal liver. Women with NAFLD had significantly higher waist circumference, BMI, AST, ALT, GGT, CHOL, TRIGL, and GLU values than women with normal liver. The levels of office SBP and DBP did not differ between the two groups. The levels of 24-hour $(P=0.011)$, daytime $(P=0.017)$, nighttime $(P=0.004)$, and morning SBP $(P=0.012)$ were higher in women with fatty liver than those without fatty liver (Table 5). In the multivariate logistic analyses, the following variables were included: BMI, essential HTN, ALT, GGT, CHOL, HDL, TRIGL, Hs-CRP, and GLU. BMI (OR: 1.277; 95\% CI:
Table 4 Variables associated with nonalcoholic fatty liver disease in men

\begin{tabular}{|c|c|c|c|c|}
\hline Variables & B & OR & $95 \% \mathrm{Cl}$ & $P$-value \\
\hline \multicolumn{5}{|l|}{ Bivariate analysis } \\
\hline Age (years) & -0.043 & 0.958 & $0.909-1.009$ & 0.104 \\
\hline Waist circumference $(\mathrm{cm})$ & $0 .|4|$ & 1.152 & $1.066-1.244$ & $<0.001$ \\
\hline $\mathrm{BMI}\left(\mathrm{kg} / \mathrm{m}^{2}\right)$ & 0.280 & 1.323 & $1.114-1.570$ & 0.001 \\
\hline Smoking (yes) & 0.235 & 1.265 & $0.39 \mid-4.097$ & 0.695 \\
\hline eGFR $\left(\mathrm{mL} / \mathrm{min} / \mathrm{l} .73 \mathrm{~m}^{2}\right)^{\mathrm{a}}$ & 0.011 & 1.011 & $0.692-1.477$ & 0.957 \\
\hline Hypertension (yes) & -0.678 & 0.508 & $0.131-1.972$ & 0.327 \\
\hline Office SBP $(\mathrm{mmHg})^{\mathrm{b}}$ & 0.679 & 1.972 & $1.972-7.668$ & 0.634 \\
\hline Office DBP (mmHg) & 0.014 & 1.014 & $0.97 \mid-1.060$ & 0.521 \\
\hline $\begin{array}{l}\text { 24-hour mean SBP } \\
(\mathrm{mmHg})\end{array}$ & 0.031 & 1.031 & $0.987-1.077$ & 0.170 \\
\hline $\begin{array}{l}\text { 24-hour mean DBP } \\
(\mathrm{mmHg})\end{array}$ & 0.010 & 1.010 & $0.959-1.063$ & 0.712 \\
\hline $\begin{array}{l}\text { Daytime mean SBP } \\
(\mathrm{mmHg})\end{array}$ & 0.021 & 1.022 & $0.98 I-1.063$ & 0.298 \\
\hline $\begin{array}{l}\text { Daytime mean DBP } \\
(\mathrm{mmHg})\end{array}$ & 0.006 & 1.006 & $0.959-1.055$ & 0.814 \\
\hline $\begin{array}{l}\text { Nighttime mean SBP } \\
(\mathrm{mmHg})\end{array}$ & 0.018 & 1.018 & $0.983-1.054$ & 0.313 \\
\hline $\begin{array}{l}\text { Nighttime mean DBP } \\
(\mathrm{mmHg})\end{array}$ & 0.010 & 1.010 & $1.038-1.059$ & 0.967 \\
\hline $\begin{array}{l}\text { Morning mean SBP } \\
(\mathrm{mmHg})\end{array}$ & 0.011 & 1.011 & $0.975-1.049$ & 0.544 \\
\hline $\begin{array}{l}\text { Morning mean DBP } \\
(\mathrm{mmHg})\end{array}$ & 0.006 & 1.006 & $0.956-1.059$ & 0.810 \\
\hline $\begin{array}{l}\text { White-coat hypertension } \\
\text { (yes) }\end{array}$ & 0.980 & 2.633 & $0.737-9.622$ & 0.295 \\
\hline AST (IU/L) & 0.060 & 1.062 & $0.987-1.142$ & 0.093 \\
\hline $\mathrm{ALT}(\mathrm{IU} / \mathrm{L})$ & 0.008 & 1.008 & $0.988-1.029$ & 0.443 \\
\hline GGT (IU/L) & 0.062 & 1.064 & $1.004-1.127$ & 0.035 \\
\hline ALP (IU/L) & -0.001 & 0.999 & $0.988-1.009$ & 0.792 \\
\hline $\mathrm{CHOL}(\mathrm{mg} / \mathrm{dL})$ & -0.007 & 0.993 & $0.980-1.006$ & 0.262 \\
\hline $\mathrm{LDL}(\mathrm{mg} / \mathrm{dL})$ & -0.010 & 0.990 & $0.975-1.005$ & 0.205 \\
\hline $\mathrm{HDL}(\mathrm{mg} / \mathrm{dL})$ & -0.025 & 0.975 & $0.943-1.009$ & 0.149 \\
\hline TRIGL (mg/dL) & 0.008 & 1.008 & $0.998-1.018$ & 0.135 \\
\hline $\mathrm{Hs}-\mathrm{CRP}(\mathrm{mg} / \mathrm{L})^{\mathrm{c}}$ & 0.371 & 1.449 & $0.503-4.172$ & 0.492 \\
\hline GLU (mg/dL) & 0.037 & 1.038 & $0.99 \mid-1.087$ & 0.119 \\
\hline \multicolumn{5}{|c|}{ Multivariate logistic regression ${ }^{\mathrm{a}}$} \\
\hline BMI $\left(\mathrm{kg} / \mathrm{m}^{2}\right)$ & 0.213 & 1.237 & $|.047-1.46|$ & 0.012 \\
\hline GGT (IU/L) & 0.055 & 1.056 & $0.991-1.127$ & 0.093 \\
\hline
\end{tabular}

Notes: Odds ratio and $95 \% \mathrm{Cl}$ adjusted by the variables with $P<0.100$ in bivariate analysis. BMI and waist circumference were not included in the same model because they are continuous variables with a correlation coefficient higher than 0.7 . The same applies to 24-hour mean SBP and daytime mean SBP, 24-hour mean SBP and nighttime mean SBP, and 24-hour mean SBP and morning mean SBP. Upper reference limits are $45 \mathrm{IU} / \mathrm{L}$ for AST, $45 \mathrm{IU} / \mathrm{L}$ for ALT, $55 \mathrm{IU} / \mathrm{L}$ for GGT, I $50 \mathrm{IU} / \mathrm{L}$ for ALP, $200 \mathrm{mg} / \mathrm{dL}$ for CHOL, $140 \mathrm{mg} / \mathrm{dL}$ for LDL, $160 \mathrm{mg} / \mathrm{dL}$ for TRIGL, $5 \mathrm{mg} / \mathrm{L}$ for $\mathrm{Hs}$-CRP, and $110 \mathrm{mg} / \mathrm{dL}$ for GLU in our laboratory. Lower reference limit is 35 $\mathrm{mg} / \mathrm{dL}$ for HDL in our laboratory. ${ }^{\text {TT }}$ The variable eGFR was transformed using square root; 'the variable office SBP was logarithmically transformed; 'the variable Hs-CRP was logarithmically transformed.

Abbreviations: $B$, regression coefficient; $\mathrm{OR}$, odds ratio; $\mathrm{Cl}$, confidence interval; BMI, body mass index; eGFR, estimated glomerular filtration rate; SBP, systolic blood pressure; DBP, diastolic blood pressure; AST, aspartate aminotransferase; ALT, alanine aminotransferase; GGT, gamma glutamyl transpeptidase; ALP, alkaline phosphatase; CHOL, cholesterol; LDL, low-density lipoprotein; HDL, high-density lipoprotein; TRIGL, triglycerides; Hs-CRP, high-density c-reactive protein; GLU, glucose serum fast. 
Table $\mathbf{5}$ Characteristics of women with and without nonalcoholic fatty liver disease

\begin{tabular}{|c|c|c|c|}
\hline Variables & $\begin{array}{l}\text { NAFLD } \\
\text { patients } \\
(\mathbf{N}=104)\end{array}$ & $\begin{array}{l}\text { Controls } \\
(\mathrm{N}=55)\end{array}$ & $P$-value \\
\hline Age (years) & $56 \pm 10$ & $55 \pm 11$ & 0.592 \\
\hline Waist circumference $(\mathrm{cm})$ & $101 \pm 13$ & $87 \pm 11$ & $<0.001$ \\
\hline BMI $\left(\mathrm{kg} / \mathrm{m}^{2}\right)$ & $30 \pm 6$ & $25 \pm 4$ & $<0.001$ \\
\hline Smoking (\%) & 23.6 & 22.0 & 0.830 \\
\hline eGFR $\left(\mathrm{mL} / \mathrm{min} / \mathrm{l} .73 \mathrm{~m}^{2}\right)$ & $84 \pm 2$ & $84.5 \pm 2$ & 0.732 \\
\hline Hypertension (\%) & 75.8 & 21.5 & 0.001 \\
\hline Office SBP (mmHg) & $146 \pm 16$ & $143 \pm 17$ & 0.226 \\
\hline Office DBP (mmHg) & $89 \pm 10$ & $87 \pm 11$ & 0.298 \\
\hline 24-hour mean SBP (mmHg) & $|29 \pm 1|$ & $124 \pm 13$ & 0.011 \\
\hline 24-hour mean DBP (mmHg) & $78 \pm 8$ & $76 \pm 9$ & 0.156 \\
\hline Daytime mean SBP $(\mathrm{mmHg})$ & $|34 \pm| \mid$ & $129 \pm 13$ & 0.017 \\
\hline Daytime mean DBP $(\mathrm{mmHg})$ & $83 \pm 9$ & $81 \pm 10$ & 0.267 \\
\hline Nighttime mean SBP $(\mathrm{mmHg})$ & $119 \pm 12$ & $111 \pm 20$ & 0.004 \\
\hline Nighttime mean DBP (mmHg) & $70 \pm 10$ & $68 \pm 10$ & 0.294 \\
\hline Morning mean SBP $(\mathrm{mmHg})$ & $130 \pm 13$ & $123 \pm 17$ & 0.012 \\
\hline Morning mean DBP $(\mathrm{mmHg})$ & $80 \pm 10$ & $77 \pm 12$ & 0.168 \\
\hline White-coat hypertension (\%) & 26 & 34.5 & 0.291 \\
\hline AST (IU/L) & $20 \pm 6$ & $19 \pm 5$ & 0.132 \\
\hline ALT (IU/L) & $23 \pm 11$ & $19 \pm 10$ & 0.018 \\
\hline GGT (IU/L) & $22 \pm 14$ & $14 \pm 6$ & $<0.001$ \\
\hline ALP (IU/L) & $103 \pm 128$ & $78 \pm 44$ & 0.183 \\
\hline $\mathrm{CHOL}(\mathrm{mg} / \mathrm{dL})$ & $220 \pm 39$ & $205 \pm 39$ & 0.031 \\
\hline LDL (mg/dL) & $138 \pm 39$ & $|3| \pm 37$ & 0.274 \\
\hline $\mathrm{HDL}(\mathrm{mg} / \mathrm{dL})$ & $61 \pm 21$ & $67 \pm 17$ & 0.084 \\
\hline TRIGL (mg/dL) & $126 \pm 57$ & $89 \pm 50$ & $<0.001$ \\
\hline $\mathrm{Hs}-\mathrm{CRP}$ (mg/L) & $1.37 \pm 2.62$ & $0.88 \pm 1.90$ & 0.283 \\
\hline GLU (mg/dL) & $94 \pm 10$ & $90 \pm 9$ & 0.019 \\
\hline
\end{tabular}

Notes: Data are presented as mean \pm standard deviation. Upper reference limits are 45 $\mathrm{IU} / \mathrm{L}$ for AST, $45 \mathrm{IU} / \mathrm{L}$ for ALT, $55 \mathrm{IU} / \mathrm{L}$ for GGT, $150 \mathrm{IU} / \mathrm{L}$ for ALP, $200 \mathrm{mg} / \mathrm{dL}$ for CHOL, $140 \mathrm{mg} / \mathrm{dL}$ for LDL, $160 \mathrm{mg} / \mathrm{dL}$ for TRIGL, $5 \mathrm{mg} / \mathrm{L}$ for Hs-CRP, and I $10 \mathrm{mg} / \mathrm{dL}$ for GLU in our laboratory. Lower reference limit is $35 \mathrm{mg} / \mathrm{dL}$ for HDL in our laboratory.

Abbreviations: NAFLD, nonalcoholic fatty liver disease; BMl, body mass index; eGFR, estimated glomerular filtration rate; SBP, systolic blood pressure; DBP, diastolic blood pressure; AST, aspartate aminotransferase; ALT, alanine aminotransferase; GGT, gamma glutamyl transpeptidase; ALP, alkaline phosphatase; CHOL, cholesterol; LDL, low-density lipoprotein; HDL, high-density lipoprotein; TRIGL, triglycerides; $\mathrm{Hs}$-CRP, high-density c-reactive protein; GLU, glucose serum fast.

1.080-1.511; $P=0.004)$ and essential HTN (OR: 6.631; 95\% CI: $1.626-27.035 ; P=0.008)$ were associated significantly with NAFLD in the multivariate logistic analyses (Table 6). BMI and waist circumference were not included in the same model due to collinearity reasons.

\section{Discussion}

The main findings of our study were that morning SBP was independently associated with the presence of NAFLD and that BMI and essential HTN were independently associated with NAFLD.

It is well known that BP increases in the morning when the subject is awake and keeps his/her daily activity.
Table 6 Variables associated with nonalcoholic fatty liver disease in women

\begin{tabular}{|c|c|c|c|c|}
\hline Variables & B & OR & $95 \% \mathrm{Cl}$ & $P$-value \\
\hline \multicolumn{5}{|l|}{ Bivariate analysis } \\
\hline Age (years) & 0.009 & 1.009 & $0.976-1.044$ & 0.589 \\
\hline Waist circumference $(\mathrm{cm})$ & 0.108 & 1.114 & $1.069-1.160$ & $<0.001$ \\
\hline BMI $\left(\mathrm{kg} / \mathrm{m}^{2}\right)$ & 0.217 & 1.242 & I.122-1.375 & 0.001 \\
\hline Smoking (yes) & 0.091 & 1.095 & $0.478-2.508$ & 0.830 \\
\hline eGFR $\left(\mathrm{mL} / \mathrm{min} / 1.73 \mathrm{~m}^{2}\right)^{\mathrm{a}}$ & -0.147 & 0.864 & $0.65 \mathrm{I}-\mathrm{I} .145$ & 0.309 \\
\hline Hypertension (yes) & 1.194 & 3.300 & $1.650-6.600$ & 0.001 \\
\hline Office SBP $(\mathrm{mmHg})^{b}$ & 1.082 & 2.950 & $0.347-3.013$ & 0.222 \\
\hline Office DBP (mmHg) & 0.017 & 1.017 & $0.985-1.050$ & 0.297 \\
\hline $\begin{array}{l}\text { 24-hour mean SBP } \\
(\mathrm{mmHg})\end{array}$ & 0.038 & 1.038 & $1.008-1.070$ & 0.013 \\
\hline $\begin{array}{l}\text { 24-hour mean DBP } \\
(\mathrm{mmHg})\end{array}$ & 0.029 & 1.030 & $0.989-1.072$ & 0.157 \\
\hline $\begin{array}{l}\text { Daytime mean SBP } \\
(\mathrm{mmHg})\end{array}$ & 0.034 & 1.035 & $1.006-1.064$ & 0.019 \\
\hline $\begin{array}{l}\text { Daytime mean DBP } \\
(\mathrm{mmHg})\end{array}$ & 0.021 & 1.021 & $0.984-1.059$ & 0.266 \\
\hline $\begin{array}{l}\text { Nighttime mean SBP } \\
(\mathrm{mmHg})\end{array}$ & 0.034 & 1.034 & $1.009-1.06 \mid$ & 0.008 \\
\hline $\begin{array}{l}\text { Nighttime mean DBP } \\
(\mathrm{mmHg})\end{array}$ & 0.018 & 1.018 & $0.984-1.053$ & 0.296 \\
\hline $\begin{array}{l}\text { Morning mean SBP } \\
(\mathrm{mmHg})\end{array}$ & 0.029 & 1.030 & $1.006-1.054$ & 0.014 \\
\hline $\begin{array}{l}\text { Morning mean DBP } \\
(\mathrm{mmHg})\end{array}$ & 0.022 & 1.022 & $0.99 \mid-1.055$ & 0.169 \\
\hline $\begin{array}{l}\text { White-coat hypertension } \\
\text { (yes) }\end{array}$ & -0.421 & 0.656 & $0.381-1.129$ & 0.128 \\
\hline AST (IU/L) & 0.052 & 1.053 & $0.984-1.128$ & 0.137 \\
\hline ALT (IU/L) & 0.051 & 1.052 & $1.007-1.100$ & 0.024 \\
\hline GGT (IU/L) & 0.098 & 1.103 & $1.042-1.166$ & 0.001 \\
\hline ALP (IU/L) & 0.005 & 1.005 & $0.997-1.014$ & 0.180 \\
\hline $\mathrm{CHOL}(\mathrm{mg} / \mathrm{dL})$ & 0.010 & 1.010 & $1.001-1.020$ & 0.035 \\
\hline $\mathrm{LDL}(\mathrm{mg} / \mathrm{dL})$ & 0.005 & 1.005 & $0.996-1.015$ & 0.273 \\
\hline $\mathrm{HDL}(\mathrm{mg} / \mathrm{dL})$ & -0.016 & 0.984 & $0.966-1.003$ & 0.092 \\
\hline TRIGL (mg/dL) & 0.018 & 1.018 & $1.008-1.028$ & $<0.001$ \\
\hline $\mathrm{Hs}-\mathrm{CRP}(\mathrm{mg} / \mathrm{dL})^{\mathrm{c}}$ & 0.625 & 1.868 & $0.916-3.806$ & 0.085 \\
\hline GLU (mg/L) & 0.044 & 1.045 & $1.006-1.085$ & 0.022 \\
\hline \multicolumn{5}{|c|}{ Multivariate logistic regression ${ }^{\mathrm{a}}$} \\
\hline BMI $\left(\mathrm{kg} / \mathrm{m}^{2}\right)$ & 0.245 & 1.277 & $1.080-1.511$ & 0.004 \\
\hline Hypertension (yes) & 1.892 & 6.631 & $1.626-27.035$ & 0.008 \\
\hline
\end{tabular}

Notes: Odds ratio and $95 \% \mathrm{Cl}$ adjusted by the variables (ALT, GGT, CHOL, HDL, TRIGL, and GLU) with $P<0.100$ in bivariate analysis. BMI and waist circumference were not included in the same model because they are continuous variables with a correlation coefficient higher than 0.7. The same applies to 24-hour mean SBP and daytime mean SBP, 24-hour mean SBP and nighttime mean SBP, and 24-hour mean SBP and morning mean SBP. Upper reference limits are $45 \mathrm{IU} / \mathrm{L}$ for AST, $45 \mathrm{IU} / \mathrm{L}$ for ALT, 55 IU/L for GGT, $150 \mathrm{IU} / \mathrm{L}$ for ALP, $200 \mathrm{mg} / \mathrm{dL}$ for CHOL, $140 \mathrm{mg} / \mathrm{dL}$ for LDL, $160 \mathrm{mg} / \mathrm{dL}$ for TRIGL, $5 \mathrm{mg} / \mathrm{L}$ for Hs-CRP, and I $10 \mathrm{mg} / \mathrm{dL}$ for GLU in our laboratory. Lower reference limit is $35 \mathrm{mg} / \mathrm{dL}$ for $\mathrm{HDL}$ in our laboratory. ${ }^{2}$ The variable eGFR was transformed using square root; ${ }^{b}$ the variable office SBP was logarithmically transformed; 'the variable $\mathrm{Hs}-\mathrm{CRP}$ was logarithmically transformed.

Abbreviations: $\mathrm{B}$, regression coefficient; $\mathrm{OR}$, odds ratio; $\mathrm{Cl}$, confidence interval; BMI, body mass index; eGFR, estimated glomerular filtration rate; SBP, systolic blood pressure; DBP, diastolic blood pressure; AST, aspartate aminotransferase; ALT, alanine aminotransferase; GGT, gamma glutamyl transpeptidase; ALP, alkaline phosphatase; CHOL, cholesterol; LDL, low-density lipoprotein; HDL, high-density lipoprotein; TRIGL, triglycerides; Hs-CRP, high-density c-reactive protein; GLU, glucose serum fast. 
This increase is accompanied with a peak incidence of myocardial infarction, sudden death, and stroke in the morning hours. ${ }^{26}$ In addition, plasma renin activity, angiotensin II, and aldosterone levels are all increased before awakening and then further increased after awakening. ${ }^{27}$ Moreover, the role of renin-angiotensin system (RAS) in the pathogenesis of arterial HTN is well known. ${ }^{28-31}$

It is documented in various scientific data that there is a local RAS within the liver, as in other tissues. Increased activation of both systemic and local RAS is involved in liver disease. The overactivation of the RAS pathway plays an important role in the pathogenesis of NAFLD. ${ }^{32,33}$ More specifically, angiotensin II leads to liver steatosis through various as yet not quite clear pathways. For example, angiotensin II leads to liver steatosis through mitochondrial oxidative damage and impaired mitochondrial betaoxidation. ${ }^{34}$

Possibly, that is the explanation of the association that was found between morning SBP and NAFLD in our study.

Essential HTN has been found associated with NAFLD by other studies. But none of them included hypertensive subjects without treatment, since growing evidence shows that angiotensin-converting-enzyme inhibitors and angiotensin II receptor blockers improve steatosis. ${ }^{13-17}$ Dixon et $\mathrm{al}^{35}$ examined severely obese patients and found that essential HTN is independently associated with advanced forms of NAFLD. The difference between our study and theirs is that their patients were under treatment for HTN, underwent liver biopsy, and $67 \%$ of them had NASH and advanced fibrosis. Bedogni et $\mathrm{al}^{12}$ in the Dionysos nutrition and liver study, found that HTN was independently associated with NAFLD. But their hypertensive population was under treatment. Vasunta et $\mathrm{al}^{11}$ showed that individuals with fatty liver had significantly higher ambulatory daytime and nighttime SBP levels. The main differences between the above study and ours are that $11.3 \%$ of their control cohort was under unknown hypertensive treatment and also $51 \%$ of the total study group was under unknown hypertensive medication.

This is the first study that included people without antihypertensive treatment. Also, we used ambulatory BP monitoring to detect essential HTN in all subjects regardless the levels of office BP. The reason all patients underwent ambulatory BP monitoring recording was that end-organ damage associated with HTN is more closely related to ambulatory BP than clinic or casual BP measurements. Additionally, ambulatory BP measurements predict the clinical outcome better when compared with office BP measurements. Ambulatory BP monitoring recordings also helped us to rule out patients with borderline or labile HTN, to exclude "white coat" HTN, and to study the whole pattern of BP during 24 hours. ${ }^{36}$

According to our results, subjects with NAFLD should be under close monitoring for their BP levels using ambulatory BP monitoring recordings.

The mechanisms associating untreated, newly diagnosed essential HTN with NAFLD are not quite clear yet. Insulin resistance is a common pathogenetic situation in NAFLD and essential HTN. Angiotensin II is also associated with the pathogenesis of insulin resistance, essential HTN, and NAFLD. ${ }^{28-37}$

Our study has two limitations. First, ultrasonography has limitations in detecting fatty liver content. Its sensitivity ranges from $82 \%$ to $89 \%$, and its specificity is approximately 93\%. Also, inflammation and fibrosis cannot be diagnosed using ultrasonography. Despite the fact that liver biopsy is the gold standard for diagnosis of NAFLD, ${ }^{38,39}$ most studies use ultrasound. ${ }^{11,12}$ Liver biopsy was not performed in this study due to ethical reasons and because no participant of our sample had the criteria to undergo liver biopsy. According to World Gastroenterology Organisation Global Guidelines of 2012, liver biopsy and histology are suitable for diagnosis of NASH when one or more of the following findings are present: abnormal serum ferritin in the absence of an elevated transferrin saturation, cytopenia, splenomegaly, clinical signs of chronic liver disease, diabetes and abnormal persistently elevated AST/ALT, obesity and age $>45$ or abnormal AST/ALT, and unexplained hepatomegaly. ${ }^{40}$

Second, the association found between HTN and NAFLD does not show causality. We took measures to strengthen the interpretation of our results (strict inclusion criteria to reduce the effect of concomitant diseases on the positive association which was found between HTN and NAFLD, covariate adjustment for anthropometric characteristics, laboratory data, and BP values). Nonetheless, the nature of the crosssectional study prevents the evaluation of the cause-effect relationship between HTN and NAFLD.

In summary, according to our findings, untreated, newly diagnosed essential HTN seems to be independently associated with the presence of NAFLD. Moreover, morning SBP is independently associated with NAFLD, whereas 24-hour and nighttime SBP showed only a tendency. Further prospective studies are needed to determine the causal relationship between essential HTN and NAFLD.

\section{Disclosure}

The authors report no conflicts of interest in this work. 


\section{References}

1. Smith BW, Adams LA. Non-alcoholic fatty liver disease. Crit Rev Clin Lab Sci. 2011;48:97-113.

2. Gossard AA, Lindor KD. Current therapies for nonalcoholic fatty liver disease. Drugs Today (Barc). 2011;47:915-922.

3. Wong VW, Wong GL, Choi PC, et al. Disease progression of nonalcoholic fatty liver disease: a prospective study with paired liver biopsies at 3 years. Gut. 2010;59:969-974.

4. Ong JP, Pitts A, Younossi ZM. Increased overall mortality and liverrelated mortality in non-alcoholic fatty liver disease. J Hepatol. 2008;49: 608-612.

5. Stepanova M, Younossi ZM. Independent association between nonalcoholic fatty liver disease and cardiovascular disease in the US population. Clin Gastroenterol Hepatol. 2012;10:646-650.

6. Nseir W, Shalata A, Marmor A, Assy N. Mechanisms linking nonalcoholic fatty liver disease with coronary artery disease. Dig Dis Sci. 2011;56(12):3439-3449.

7. Lizardi-Cervera J, Aguilar-Zapata D. Nonalcoholic fatty liver disease and its association with cardiovascular disease. Ann Hepatol. 2009;8(Supp1 1):S40-S43.

8. Gariani K, Philippe J, Jornayvaz FR. Non-alcoholic fatty liver disease and insulin resistance: from bench to bedside. Diabetes Metab. 2013;39: 16-26.

9. Marchesini G, Bugianesi E, Forlani G, et al. Nonalcoholic fatty liver, steatohepatitis, and the metabolic syndrome. Hepatology. 2003;37: 917-923.

10. Marchesini G, Marzocchi R. Metabolic syndrome and NASH. Clin Liver Dis. 2007;11:105-117.

11. Vasunta RL, Kesäniemi YA, Ylitalo AS, Ukkola OH. High ambulatory blood pressure values associated with non-alcoholic fatty liver in middle-aged adults. J Hypertens. 2012;30:2015-2019.

12. Bedogni G, Miglioli L, Masutti F, Tiribelli C, Marchesini G, Bellentani S. Prevalence of and risk factors for nonalcoholic fatty liver disease: the Dionysos nutrition and liver study. Hepatology. 2005;42:44-52.

13. Georgescu EF. Angiotensin receptor blockers in the treatment of NASH/NAFLD: could they be a first-class option? Adv Ther. 2008;25: 1141-1174.

14. Fogari R, Maffioli P, Mugellini A, Zoppi A, Lazzari P, Derosa G. Effects of losartan and amlodipine alone or combined with simvastatin in hypertensive patients with nonalcoholic hepatic steatosis. Eur J Gastroenterol Hepatol. 2012;24:164-171.

15. Yamamoto E, Dong YF, Kataoka K, et al. Olmesartan ameliorates a dietary rat model of non-alcoholic steatohepatitis regulating kinase-1 inhibition. Eur J Pharmacol. 2008;588(2-3):316-324.

16. Kitamura K, Tada S, Nakamoto N, et al. Rho/Rho kinase is a key enzyme system involved in the angiotensin II signaling pathway of liver fibrosis and steatosis. J Gastroenterol Hepatol. 2007;22(11):2022-2033.

17. Kurita S, Takamura T, Ota T, et al. Olmesartan ameliorates a dietary rat model of non-alcoholic steatohepatitis through its pleiotropic effects. Eur J Pharmacol. 2008;588(2-3):316-324.

18. Lee BT, Ahmed FA, Hamm LL, et al. Association of C-reactive protein, tumor necrosis factor-alpha, and interleukin-6 with chronic kidney disease. BMC Nephrol. 2015;16:77.

19. Angulo P. Medical progress: nonalcoholic fatty liver disease. $N$ Eng $J$ Med. 2002;346:1221-1231.

20. Park SH, Kim BI, Kim SH, Kim HJ, Park DI, Cho YK. Body fat distribution and insulin resistance: beyond obesity in nonalcoholic fatty liver disease among overweight men. J Am Coll Nutr. 2007;26:321-326.

21. Douketis JD, Paradis G, Keller H, Martineau C. Canadian guidelines for body weight classification in adults: application in clinical practice to screen for overweight and obesity and to assess disease risk. CMAJ. 2005;172(8):995-998

22. Sanyal AJ; for American Gastroenterological Association. AGA technical review on nonalcoholic fatty liver disease. Gastroenterology. 2002;123(5):1705-1725.
23. ESH/ESC Task Force for the Management of Arterial Hypertension. 2013 Practice guidelines for the management of arterial hypertension of the European Society of Hypertension (ESH) and the European Society of Cardiology (ESC): ESH/ESC Task Force for the Management of Arterial Hypertension. J Hypertens. 2013;31(10):1925-1938.

24. O'Brien E. Twenty-four-hour ambulatory blood pressure measurement in clinical practice and research: a critical review of a technique in need of implementation. J Intern Med. 2011;269(5):478-495.

25. O'Brien E, Parati G, Stergiou G, et al; European Society of Hypertension Working Group on Blood Pressure Monitoring. European Society of Hypertension position paper on ambulatory blood pressure monitoring. J Hypertens. 2013;31(9):1731-1768.

26. Flack JM, Yunis C. Therapeutic implications of the epidemiology and timing of myocardial infarction and other cardiovascular diseases. J Hum Hypertens. 1997;11(1):23-28.

27. Brandenberger G, Follenius M, Goichot B, et al. Twenty-four-hour profiles of plasma renin activity in relation to the sleep-wake cycle. J Hypertens. 1994;12(3):277-283.

28. Zhou MS, Schulman IH, Raij L. Role of angiotensin II and oxidative stress in vascular insulin resistance linked to hypertension. Am J Physiol Heart Circ Physiol. 2009;296:H833-H839.

29. Te Riet L, van Esch JH, Roks AJ, van den Meiracker AH, Danser AH. Hypertension: renin-angiotensin-aldosterone system alterations. Circ Res. 2015;116(6):960-975.

30. Moon JY. Recent update of renin-angiotensin-aldosterone system in the pathogenesis of hypertension. Electrolyte Blood Press. 2013; $11(2): 41-45$.

31. Manrique C, Lastra G, Gardner M, Sowers JR. The renin angiotensin aldosterone system in hypertension: roles of insulin resistance and oxidative stress. Med Clin North Am. 2009;93(3):569-582.

32. Frantz ED, Penna-de-Carvalho A, Batista Tde M, Aguila MB, Mandarim-de-Lacerda CA. Comparative effects of the renin-angiotensin system blockers on nonalcoholic fatty liver disease and insulin resistance in C57BL/6 mice. Metab Syndr Relat Disord. 2014;12(4):191-201.

33. Paschos P, Tziomalos K. Nonalcoholic fatty liver disease and the reninangiotensin system: implications for treatment. World J Hepatol. 2012; 4(12):327-331.

34. Wei Y, Clark SE, Thyfault JP, et al. Oxidative stress-mediated mitochondrial dysfunction contributes to angiotensin II-induced nonalcoholic fatty liver disease in transgenic Ren2 rats. Am J Pathol. 2009; 174(4):1329-1337.

35. Dixon JB, Bhathal PS, O'Brien PE. Nonalcoholic fatty liver disease: predictors of nonalcoholic steatohepatitis and liver fibrosis in the severely obese. Gastroenterology. 2001;121(1):91-100.

36. McGrath BP; for National Blood Pressure Advisory Committee of the National Heart Foundation of Australia. Ambulatory blood pressure monitoring. Med J Aust. 2002;176(12):588-592.

37. Matthew Morris E, Fletcher JA, Thyfault JP, Scott Rector R. The role of angiotensin II in nonalcoholic steatohepatitis. Mol Cell Endocrinol. 2013;378(1-2):29-40.

38. Joseph AE, Saverymuttu SH, Al-Sam S, Cook MG, Maxwell JD. Comparison of liver histology with ultrasonography in assessing diffuse parenchymal liver disease. Clin Radiol. 1991;43:26-31.

39. Hultcrantz R, Gabrielsson N. Patients with persistent elevation of aminotransferases: investigation with ultrasonography, radionuclide imaging and liver biopsy. J Intern Med. 1993;233:7-12.

40. LaBrecque D, Abbas Z, Anania F, et al. WGO Global Guidelines Library c2006-2015. Nonalcoholic Fatty Liver Disease and Nonalcoholic Steatohepatitis. Milwaukee, WI: World Gastroenterology Organisation; June 2012. Available from: http://www.worldgastroenterology.org/ assets/export/userfiles/2012_NASH\%20and\%20NAFLD_Final_long. pdf. Accessed November 19, 2015. 
Clinical and Experimental Gastroenterology

Dovepress

\section{Publish your work in this journal}

Clinical and Experimental Gastroenterology is an international, peerreviewed, open access journal, publishing all aspects of gastroenterology in the clinic and laboratory, including: Pathology, pathophysiology of gastrointestinal disease; Investigation and treatment of gastointestinal disease; Pharmacology of drugs used in the alimentary tract;

Immunology/genetics/genomics related to gastrointestinal disease. This journal is indexed on CAS. The manuscript management system is completely online and includes a very quick and fair peer-review system. Visit http://www.dovepress.com/testimonials.php to read real quotes from published authors.

Submit your manuscript here: http://www.dovepress.com/clinical-and-experimental-gastroenterology-journal 